\title{
Componente hereditario materno y paterno en el riesgo de preeclampsia
}

Paternal and maternal components of the predisposition to preeclampsia. Esplin MS, Bardett Fausett M, Fraser A y col. N Engl J Med 2001 Mar 22; 344:867-72.

\section{Objetivo}

Determinar si las mujeres o los hombres cuyas madres tuvieron preeclampsia durante su embarazo tienen mayor riesgo de tener hijos de un embarazo complicado con preeclampsia

\section{Diseño}

Estudio de cohorte. Se evaluaron 2 grupos: Los hombres o mujeres expuestos - sujetos cuyas madres habían tenido preeclampsia- fueron apareados aleatoriamente a dos controles cuyas madres no la habían padecido, según sexo, edad materna, año de nacimiento, orden de nacimiento y jurisdicción de nacimiento en Utah.

\section{Lugar}

Utah, EE.UU.

\section{Fuente de datos}

Los datos fueron obtenidos de la base de datos de Utah, diseñada para evaluar predisposición familiar a complicaciones del embarazo.

\section{Participantes}

Se identificaron como expuestos, 298 hombres y 237 mujeres quienes durante un periodo de estudio de 23 años habían tenido 947 y 830 hijos respectivamente. El grupo control quedó conformado por 596 hombres y 474 mujeres quienes para el mismo periodo habían tenido 1973 y 1658 hijos respectivamente.

Fueron excluidos aquellos sujetos cuyas madres tenían complicaciones médicas preexistentes o complicaciones del embarazo que se asociaran con preeclampsia

\section{Medición de resultados principales}

El resultado principal evaluado fue tener un hijo producto de un embarazo complicado con preeclampsia Para ambos grupos se tuvo en cuenta el registro diagnóstico de enfermedad especificado en el certificado de nacimiento y volcado en la base de datos.

\section{Evaluación de factores de riesgo}

Se evaluaron 15 variables de riesgo y su posible efecto confundidor: sexo fetal, edad materna y paterna, año de nacimiento, área de residencia, raza materna y paterna, paridad materna, número de recién nacidos vivos, número de pérdidas de embarazos, nivel de educación materna y paterna, estado civil, edad gestacional al nacer, y orden de nacimiento. Se efectuo un analisis de regresión logística para determinar el efecto independiente de cada una de las variables estudiadas.

\section{Resultados principales}

Las mujeres y los hombres expuestos tuvieron un mayor riesgo de tener hijos producto de un embarazo con preeclampsia respecto de Ios controles: odds ratio hombres, 2.1 ; IC $95 \%$ [1.0 a 4.3], $p=0.04$. odds ratio mujeres: $3.3 ;$ [1.5 a 7.5] $p=0.004$

\begin{tabular}{l|l|l|l|l}
\hline & $\begin{array}{l}\text { Hombres } \\
\text { (estudio) }\end{array}$ & $\begin{array}{l}\text { Hombres } \\
\text { (control) }\end{array}$ & $\begin{array}{l}\text { Mujeres } \\
\text { (estudio) }\end{array}$ & $\begin{array}{l}\text { Mujeres } \\
\text { (conlrol) }\end{array}$ \\
\hline $\mathrm{N}$ total & 298 & 596 & 237 & 474 \\
\hline $\mathrm{N}^{0}$ de hijos & 947 & 1973 & 830 & 1658 \\
\hline $\begin{array}{l}\text { Hijos producto } \\
\text { de un embarazo } \\
\text { con preeclampsia }\end{array}$ & $26(2.7 \%)$ & $26(1.3 \%)$ & $39(4.7 \%)$ & $32(1.9 \%)$ \\
\hline
\end{tabular}

El riesgo de preeclampsia se asocio tambien a nuliparidad y pretérmino.

\section{Conclusión}

Las mujeres cuyas madres habían tenido preeclampsia tienen mayor riesgo de tener preeclampsia durante el embarazo respecto de las no expuestas. El mismo efecto aunque mas débil se observó en los hombres.

\section{Comentario}

La preeclampsia se presenta aproximadamente en el $5 \%$ de los embarazos, y es la $2^{\circ}$ causa de muerte materna en Estados Unidos y la $3^{\circ}$ en Argentina La etiología es desconocida y se proponen diversos mecanismos fisiopatológicos, entre los cuales se describen mecanismos inmunológicos y predisposición genética.

En este estudio se observa que en aquellas mujeres el antecedente materno incrementa 3 veces el riesgo de padecer la enfermedad durante el embarazo. Con respecto al riesgo aportado por el antecedente paterno existe una tendencia en el mismo sentido, pero se necesitaría un tamaño muestral más grande para demostrar una diferencia significativa.

Este estudio cuenta con aspectos metodológicos que lo hacen fuerte: es un estudio de cohorte en donde se excluyeron aquellos sujetos con los principales factores de riesgo conocidos para preeclampsia, siendo ambos grupos estudiados similares en sus características. Por otra parte, por la naturaleza de la base de datos evita el sesgo de memoria habitualmente observados en estudios a largo plazo y el sesgo del entrevistador.

Sin embargo, tambien por la forma de recolección de los datos, aspectos clínicos asociados a la preeclampsia que pudieran actuar como variables de confusión no han podido ser controlados 1,2. Por otra parte, no se clasificó la preeclampsia según gravedad que para algunos autores tendría componentes hereditarios diferentes 3

Este estudio coincide con la literatura sobre el factor de riesgo que implica el antecedente materno en las mujeres embarazadas. Lo mismo ocurre con algunos trabajos que mencionan el antecedente paterno, evaluado tanto en animales como en humanos, así como la fisiopatología que justificaría esta evidencia.3,4

En conclusión, el presente estudio podría ser aplicado a nuestra población, y dado sus resultados y la coincidencia con estudios publicados anteriormente, se cuenta con evidencia suficiente para considerar un patrón hereditario de la preeclampsia y generar nuevas hipótesis con el fin de demostrar más claramente la predisposición genética de esta enfermedad.

\section{Dr. Glujovsky, Demian - Dr. Krupitzki, Hugo [ Departamento de Ginecología y Obstetricia. Dirección de Investigación. CEMIC ]}

\section{Referencias}

1. Lie RT, Rasmussen S, Brunborg H, et al. Fetal and maternal contributions to risk of pre-eclampsia: population based study. BMJ 1998 May 2:316(7141):1343-7. 2. Arngrimsson R, Bjornsson S, Geirsson RT, Bjornsson H, Walker JJ, Snaedal G. Genetic and familial predisposition to eclampsia and pre-eclampsia in a defined population. Br J Obstet Gynaecol 1990;97:762769

3. Mogren I, Hogberg U, Winkvist A, Stenlund H. Familial occurrence of preeclampsia. Epidemiology 1999;10:518-522.

4. Dekker G, Sibai B. Etiology and pathogenesis of preeclampsia: Current concepts. Am J Obstet Gynecol 1998;179:1359-75. 